\title{
Lucrèce et la tradition de la consolation
}

\section{Sabine Luciani}

\section{(2) OpenEdition}

Journals

Édition électronique

URL : https://journals.openedition.org/rhetorique/519

DOI : $10.4000 /$ rhetorique.519

ISSN : 2270-6909

\section{Éditeur}

UGA Éditions/Université Grenoble Alpes

\section{Édition imprimée}

ISBN : 978-2-37747-010-5

\section{Référence électronique}

Sabine Luciani, « Lucrèce et la tradition de la consolation », Exercices de rhétorique [En ligne], 9 | 2017 , mis en ligne le 20 juin 2017, consulté le 21 septembre 2021. URL : http://journals.openedition.org/ rhetorique/519 ; DOI : https://doi.org/10.4000/rhetorique.519

Ce document a été généré automatiquement le 21 septembre 2021.

\section{(c) (i) (2)(2)}

Les contenus de la revue Exercices de rhétorique sont mis à disposition selon les termes de la Licence Creative Commons Attribution - Pas d'Utilisation Commerciale - Partage dans les Mêmes Conditions 4.0 International. 


\title{
Lucrèce et la tradition de la consolation
}

\author{
Sabine Luciani
}

1 «Le poème de Lucrèce est en un sens une consolation. Il l'est par son but, par sa préoccupation constante de délivrer les hommes de la crainte de la mort, en montrant que la mort n'est pas un mal. Il ne l'est à aucun point par sa méthode. Appliquant une thérapeutique morale d'une valeur tout empirique, la consolation cherche à créer, par l'exercice mental qu'est la méditation, et au besoin par la simple répétition des mêmes motifs et des mêmes formules, un certain état d'esprit ; elle se propose de modifier les dispositions du sujet par une pénétration progressive et une sorte de transmutation. C'est, au contraire, sur une démonstration scientifique que Lucrèce prétend fonder une conviction logique et raisonnée ${ }^{1}$ ». Si l'on en croit ce jugement formulé en 1940 par Paul Vallette, le De rerum natura ne peut être rattaché au genre de la consolation parce que Lucrèce y expose de façon rigoureuse et argumentée les principes de la doctrine épicurienne, dont il veut mettre en évidence la validité théorique et l'exactitude scientifique ${ }^{2}$. Pourtant il est largement admis désormais que Lucrèce, pour transmettre à Rome la doctrine d'Épicure et guider les lecteurs sur la voie de l'ataraxie ${ }^{3}$, combine précisément à la rigueur doctrinale les outils de persuasion fournis par la rhétorique, qui sont mis au service du message épicurien ${ }^{4}$. Il ne paraît pas nécessaire de s'étendre sur ce point.

2 En revanche, l'opposition établie entre rationalité et consolation trouve un écho dans les questionnements actuels sur la consolation : dans un livre récent, Michaël Fœssel oppose à la philosophie moderne, qui «a abandonné la consolation, d'une part à la religion, de l'autre à la psychologie», la philosophie antique pour laquelle «la consolation était une prérogative de la raison [...] boussole infaillible, non seulement pour éviter le pire, mais pour l'affronter lorsqu'il est advenu ${ }^{5}$ ». Cette antithèse structurante, qui permet à l'auteur d'élaborer une réflexion originale sur le désir de consolation et le pouvoir subversif du chagrin, comporte une indéniable valeur heuristique. Et l'on ne peut qu'être globalement d'accord avec la thèse selon laquelle les penseurs antiques voyaient dans le savoir rationnel « une arme contre les péripéties de 
l'existence ». Cependant, aussi séduisante soit-elle, cette présentation binaire, qui vise prioritairement à étayer une analyse du désenchantement moderne, tend à donner une image trop schématique de la consolation antique.

3 Face à ces deux visions contradictoires, le présent article se propose d'examiner pour elles-mêmes les relations entre consolation et rationalité philosophique à l'aune du texte lucrétien. La crainte de la mort constitue en effet la cible principale du poète, qui entend procurer à son disciple Memmius un soulagement face aux maux inhérents à la condition humaine et un réconfort contre les aléas de la fortune. C'est pourquoi il développe, au troisième chant du poème, une ample méditation sur la mort, fondée sur l'opposition entre la réalité physiologique d'une dissolution atomique (III, 93-829) et les angoisses suscitées par de vains préjugés (III, 830-10936). Dans ce cadre, comme l'a souligné J. Salem, l'argumentation fait fond sur les motifs et les procédés issus de la littérature consolatoire ${ }^{7}$. Mais, le De rerum natura, qui se veut d'abord et avant tout un exposé scientifique de la doctrine épicurienne, peut-il pour autant prétendre au titre de consolation? Cette question est totalement négligée dans les ouvrages publiés sur le sujet par C. Alonso Del Real, F. Lillo Redonet et H. Baltussen, dans lesquels ni l'éthique épicurienne ni le poème de Lucrèce en particulier ne font l'objet d'analyses spécifiques ${ }^{8}$. Exceptée une remarquable étude de D. Konstan consacrée à la thérapie épicurienne du chagrin ${ }^{9}$, la question ne retient pas non plus l'attention des spécialistes de l'éthique épicurienne, dont l'intérêt tend actuellement à se focaliser sur le statut des émotions ${ }^{10}$.

Dans ce contexte, il ne sera pas inutile de mettre à profit ces travaux récents pour revenir sur les relations entre la consolation en tant que genre littéraire reflétant un ensemble de discours et de pratiques sociales et le projet lucrétien en tant que texte philosophico-poétique. Afin de déterminer dans quelle mesure le De rerum natura se rattache au genre de la consolation, nous tenterons de faire une mise au point sur les formes prises par la consolation antique et sur les rapports qu'elle entretient avec la philosophie en général et l'épicurisme en particulier.

\section{Formes et signification de la consolation antique}

En formulant une opposition entre, d'une part, la consolation, qui viserait exclusivement à soulager les affligés par la persuasion, et d'autre part, la philosophie, dont l'objectif serait de convaincre le disciple par une argumentation rationnelle, Paul Vallette cherchait à démontrer que, dans la deuxième partie du chant III, Lucrèce s'était inspiré d'une source non-épicurienne. Fondant son raisonnement sur une communauté générique entre diatribe et consolation, il voyait dans la tonalité rhétorique des vers consacrés à la réfutation de la crainte de la mort (DRN, III, 830-1094) une marque de dépendance à l'égard des prédications morales de type populaire pratiquées par les philosophes cyniques et stoïciens ${ }^{11}$. Cependant, la définition et l'existence même de la diatribe antique, en tant que genre littéraire constitué, sont désormais controversées ${ }^{12}$. De plus, même si Lucrèce a pu s'inspirer des procédés utilisés dans les diatribes, comme le dialogisme ou la prosopopée ${ }^{13}$, l'influence de cette tradition, elle-même nourrie de lieux communs, ne doit pas être surévaluée ${ }^{14}$. Enfin, les emprunts lucrétiens aux différents genres littéraires, dans la mesure où ils sont placés au service d'un projet philosophique, n'impliquent pas de distorsion par rapport à l'enseignement épicurien. En dépit de ces difficultés, l'opposition établie entre les 
méthodes de la consolation (et de la diatribe) et celles de la philosophie n'en est pas pour autant dépassée. Elle renvoie en effet à plusieurs questions ayant trait, d'une part, aux contours du genre consolatoire et, d'autre part, à ses relations complexes avec la philosophie.

\section{Un genre polymorphe}

6 Si d'Homère à Libanios, les topiques consolatoires occupent une place importante dans la littérature antique, la consolation, en tant que genre, pose des problèmes de définition. Depuis les consultations dispensées aux affligés par l'orateur Antiphon ${ }^{15}$ sur l'agora de Corinthe au V $\mathrm{V}^{\mathrm{e}}$ siècle avant notre ère jusqu'à la Consolation de Philosophie composée par Boèce à la fin de l'Antiquité, elle se développa en effet selon des formes, des méthodes et des intentions extrêmement variées. Conjointement pratiquée par les orateurs, les philosophes et les poètes, elle pouvait prendre la forme de lettres, de discours, de traités aussi bien que de poèmes. Même si une grande partie de ces écrits est aujourd'hui perdue, de nombreux témoignages attestent l'ampleur de leur développement à partir de l'époque hellénistique ${ }^{16}$. Outre les écrits explicitement consolatoires composés par Cicéron, Sénèque, Plutarque et Pline le Jeune ${ }^{17}$, plusieurs poèmes, notamment une épigramme de Catulle et deux Odes d'Horace ${ }^{18}$, peuvent prétendre au titre de consolation ${ }^{19}$. Cependant, en dépit de sa large diffusion, cette pratique littéraire ne fut que tardivement théorisée et, au premier siècle avant notre ère, Cicéron soulignait qu'on ne trouvait dans les écrits des rhéteurs aucun précepte sur la consolation ${ }^{20}$. La codification du genre est le fait des écoles de rhétorique d'époque impériale, dans lesquelles la consolation faisait partie des exercices proposés aux élèves ${ }^{21}$. Dans ce contexte, le discours consolatoire, logos paramuthètikos, est évoqué dans plusieurs manuels en relation avec l'oraison funèbre, logos epitaphios, dont il constitue la partie finale ${ }^{22}$. Chez le Pseudo-Démétrios, le champ de la consolation, qui est envisagée dans le cadre d'une typologie épistolaire, est étendu à tous les maux qui affectent le genre humain : la lettre de réconfort est «celle qu'on écrit à des gens frappés d'une peine à la suite de quelque événement désagréable ${ }^{23} »$.

\section{Typologies}

7 Si l'on se réfère à ces catégories et aux différents exemples cités, la consolation peut à bon droit être définie comme un écrit ou un discours visant « à dissiper ou du moins à modérer, autant que possible, le chagrin suscité par les événements malheureux ou considérés comme malheureux selon l'opinion courante (décès, maladie, exil, vieillesse

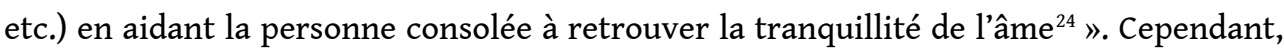
pour être nuancée et précise, une telle définition pourra aussi sembler restrictive, en ce qu'elle tend à limiter le corpus à des textes de circonstance. Elle ne prend pas en compte les écrits moraux qui se proposent non seulement d'aider les lecteurs à supporter, le cas échant, un chagrin actuel, mais de les soulager de tous les maux inhérents à la condition humaine. On peut citer dans cette veine le traité de Chrysippe Sur les passions ${ }^{25}$, celui (perdu) du stoïcien Panetius Sur la douleur ${ }^{26}$, les Tusculanes de Cicéron $^{27}$, ou encore, à l'époque impériale, les traités de Sénèque Sur la tranquillité de l'âme ou Sur la mort prématurée, ceux de Plutarque Sur l'exil ou Sur la tranquillité de l'âme. Le statut particulier de ces écrits et les liens distendus qu'ils entretiennent au genre de la consolation ont conduit R. Kassel, auteur d'une importante étude sur la consolation 
publiée en 1958, à opérer une distinction entre consolation au sens strict et consolation au sens large ${ }^{28}$ : stricto sensu, la consolation est un écrit - le plus souvent une lettre composé à l'occasion d'un deuil pour apaiser le chagrin de l'endeuillé. Au sens large, la consolation est un écrit composé en dehors de circonstances précises et immédiates, et qui vise à fournir des remèdes contre les divers maux de la vie ${ }^{29}$. Cependant, bien qu'elle attire à juste titre l'attention sur la forme personnelle inhérente à ce type de textes, cette opposition, concentrée sur le deuil, ne tient pas suffisamment compte de la variété des situations à traiter, que ce soit au plan individuel (exil, maladie, douleur, cécité) ou au plan collectif (défaite militaire, ruine de cité, épidémie, réduction en esclavage). D'autre part, elle ne permet pas d'envisager la dimension réflexive de certains textes comme les Tusculanes, qui s'interrogent sur les fondements théoriques des différentes topiques et sur les conditions d'efficacité des méthodes mises en œuvre ${ }^{30}$.

8 C'est pourquoi, nous privilégierons la typologie élaborée par David Scourfield, qui propose d'envisager prioritairement la consolation en relation aux pratiques sociales ${ }^{31}$. En référence à ce modèle fonctionnel, il suggère de réserver le terme générique de consolation aux consolations effectives, adressées à un (ou plusieurs) destinataire(s) spécifique(s) dans une situation particulière. Par ailleurs, il introduit le terme de "méta-consolation" pour désigner des textes conçus sur le mode réflexif, qui constituent un réservoir d'arguments à utiliser ou présentent une analyse des méthodes et des pratiques consolatoires. Cette division est fort éclairante en ce qu'elle permet de penser plus précisément l'articulation entre philosophie et consolation.

\section{Consolation et philosophie}

9 En dépit de ses origines sophistiques, la consolation fit rapidement son entrée dans le champ de la philosophie et devint une composante importante des différentes éthiques hellénistiques. Le mérite d'avoir composé, au tournant du III siècle avant notre ère, la première consolation philosophique est communément attribué au philosophe académicien Crantor de Soles, dont le fameux traité sur le deuil, Peri penthous, était considéré comme un modèle du genre ${ }^{32}$. Même si cet ouvrage n'a pas été conservé, l'importance qui lui est attribuée dans l'histoire de la philosophie témoigne de l'attention portée par les écoles hellénistiques à l'articulation entre logos et consolation. Soucieux de conduire leurs disciples sur la voie du bonheur, les philosophes cherchaient à les guérir des troubles qui, assaillant perpétuellement leur âme, les éloignaient de la sérénité. Or, parmi ces troubles de l'âme à éradiquer, figure en première place le chagrin, dont le deuil constitue la forme la plus terrible ${ }^{33}$. Dans cette perspective, s'est développée une vaste réflexion éthique sur la vocation consolatoire de la philosophie, en tant que médecine de l'âme ${ }^{34}$. Cependant, contrairement à ce que pourraient suggérer le cas de Cantor et les différents exemples cités, la consolation, pour avoir été pratiquée au sein de l'Académie et du Portique, n'en n'a pas été pour autant négligée au sein du Jardin. Et si l'on en croit le témoignage de Plutarque, Épicure a pris parti dans la querelle philosophique autour de la thérapie des passions $^{35}$. Face aux stoïciens qui prônaient l'éradication totale du chagrin et de toutes les passions, il voulait qu'on se contentât de les limiter, à l'instar des péripatéticiens et de Crantor $^{36}$. 


\section{Consolations épicuriennes}

10 Rudolf Kassel avait déjà noté que la consolation jouait un rôle important dans l'éthique épicurienne ${ }^{37}$. Le savant allemand fondait son affirmation sur plusieurs éléments : outre le caractère individuel et le ton personnel des lettres adressées par Épicure à ses disciples et, de manière générale, la place accordée à l'amitié au sein du Jardin, il mentionnait notamment le caractère thérapeutique des quatre premières Maximes Capitales tendues vers l'ataraxie ${ }^{38}$ et la Sentence vaticane 55 , qui définit les principes d'une méthode contre le chagrin, fondée sur «la reconnaissance pour ce qu'y est perdu » et la conscience « qu'il n'est pas possible de faire que ce qui a été ne se soit pas accompli ${ }^{39} »$. Par ailleurs, on sait par Plutarque qu'Épicure, après le décès de son disciple Hégésianax, avait écrit des lettres de consolation au père et au frère de celui$\mathrm{ci}^{40}$. Plutarque indique également à cette occasion que le fondateur du Jardin, critiquant l'insensibilité prônée par les stoïciens, considérait qu'il fallait laisser libre cours au chagrin et aux larmes provoqués par la mort d'un être cher. Estimant que le sage était, plus que les autres hommes, sujet aux émotions ${ }^{41}$, il conseillait même, suivi en cela par son disciple Métrodore, de rechercher cet étrange plaisir qui se mêle aux larmes du deuil ${ }^{42}$.

11 Ce faisceau d'éléments convergents a incité D. Konstan ${ }^{43}$ à voir dans Épicure, dont le nom même renvoie à l'idée de secours, l'inventeur de la consolation philosophique, alors que ce mérite est communément attribué au philosophe académicien Crantor de Soles $^{44}$. Bien que cette hypothèse soit actuellement difficile à démontrer compte tenu $\mathrm{du}$ caractère partiel et fragmentaire des textes conservés, elle attire du moins l'attention sur la visée consolatrice de l'enseignement épicurien, centré sur la thèse selon laquelle « la mort n'est rien pour nous ${ }^{45} »$. De fait, comme le souligne Ph. Mitsis, «il revient aux épicuriens d'avoir maintenu avec le plus de véhémence que c'est une thanatologie qui doit fournir l'axe central de toute théorie éthique véritablement convaincante ${ }^{46} »$. Nous n'avons malheureusement pas conservé de lettre de condoléances épicurienne, mais le traité De morte composé par le Syrien Philodème de Gadara, contemporain de Cicéron, dont les œuvres ont été partiellement conservées dans la "villa des papyri » à Herculanum, montre à tout le moins que la question du deuil et de l'attitude face à la mort fut une préoccupation constante au sein de l'école ${ }^{47}$. Puisque «nous habitons tous une cité sans remparts contre la mort » (De morte, col. 37), le philosophe, tout en reconnaissant le caractère naturel des émotions éprouvées face à notre propre mort ou à celle des êtres qui nous sont chers, adresse à ses lecteurs des conseils pour se préparer à mourir sereinement et/ou surmonter la peine provoquée par la perte d'un être cher. Pour ce faire, il oppose à l'attitude des insensés qui chassent la pensée de la mort (col. 38), la conduite des sages qui « rendent leur dernier souffle sans aucune frayeur » (col. 39). Par conséquent, bien que ce traité ne corresponde pas, pour autant qu'on puisse le savoir, à une consolation spécifique, il entre néanmoins dans la catégorie des méta-consolations: plusieurs cas particuliers et exemples pratiques y sont envisagés, dans une perspective générale, en relation aux conditions et aux méthodes qui conduisent à la tranquillité de l'âme.

De son côté, le De rerum natura, qui a été composé à peu près à la même époque par le poète Lucrèce ${ }^{48}$, donne lieu à des interprétations radicalement différentes. Et le point de vue de Paul Vallette, qui exclut résolument le poème du champ de la consolation, est encore largement partagé. 


\section{Lucrèce vs Épicure}

arguments invoqués pour réfuter l'orientation consolatoire du De rerum natura résident prioritairement dans une opposition entre la sévérité dont fait preuve Lucrèce à l'égard de ceux qui craignent la mort et l'indulgence compréhensive dont témoignent les écrits d'Épicure et de Philodème ${ }^{49}$. Cette antithèse a été ravivée par les études récentes consacrées au statut des émotions dans l'épicurisme, et plus particulièrement dans les traités de Philodème ${ }^{50}$. Il a notamment été démontré que le philosophe épicurien insistait sur le caractère naturel de certaines émotions que le sage ressent dans certaines circonstances ${ }^{51}$. Il semble en effet que, sans remettre en cause la possibilité d'atteindre la sérénité, Philodème ait fait preuve d'une certaine tolérance à l'égard d'affects comme la colère ou le chagrin et notamment considéré comme légitimes les larmes versées suite à la perte d'un être cher. Face à de telles concessions, qui sont par ailleurs conformes aux témoignages antiques concernant la position d'Épicure ${ }^{52}$, les propos que Lucrèce attribue à la Nature dans la célèbre prosopopée du chant III (v. 931-962) se signalent par leur extrême intransigeance ${ }^{53}$. S'adressant à un vieillard qui refuse de mourir, la Nature le réprimande sur un ton dépourvu d'aménité (uoce increpet acri, DRN, III, 956 ${ }^{54}$ ) :

Arrête-moi ces pleurs, gouffre béant, ravale ces plaintes !

Les biens de la vie tous épuisés, te voilà décrépit.

Mais à toujours vouloir ce que tu n'as pas,

à mépriser ce que tu as, la vie s'est écoulée,

incomplète et sans joie, et soudain tu t'étonnes :

La mort s'est installée à ton chevet

avant que tu ne puisses prendre congé du monde,

Content et rassasiés5.

Aufer abhinc lacrimas, barathre, et compesce querellas.

omnia perfunctus uitai praemia marces ;

sed quia semper aues quod abest, praesentia temnis,

inperfecta tibi elapsast ingrataque uita,

et nec opinanti mors ad caput adstitit ante

quam satur ac plenus possis discedere rerum. (DRN, III, 955-960)

Le discours s'achève par une injonction pressante à laisser la place (v. 961-963). Cette condamnation sans appel paraît fort éloignée de la compassion qu'un consolateur est censé témoigner à son destinataire ${ }^{56}$. Arguant de cette tonalité véhémente et de la structure du poème orientée vers la réfutation des craintes humaines, A. D. Morrison considère que la consolation n'est pas l'objectif de Lucrèce et insiste sur "l'extrémisme » éthique du poète, qui rejetterait l'empathie et la consolation pratiquées par les successeurs du maître ${ }^{57}$. Outre le fait qu'Épicure semble avoir parfois assumé le rôle de consolateur, cette analyse, qui repose sur une vision schématique de la consolation antique, ne parait pas convaincante car elle ne prend pas suffisamment en compte les intentions du poète.

\section{Le De rerum natura et le genre consolatoire}

Plusieurs éléments d'ordre lexical, structurel et méthodologiques indiquent que le poète a délibérément inscrit son projet dans la tradition de la consolation. 


\section{Lexique}

Force est de constater que ni le substantif consolatio et ni le verbe consolari ne figurent dans le poème. Si l'absence du substantif est liée à la métrique dactylique, cette raison ne vaut pas pour le verbe, qui est fréquemment employé en poésie ${ }^{58}$. Pourtant cette lacune est palliée par 4 occurrences du terme solacium, et en particulier de l'expression solacia uitae, qui est employée dans deux passages-clés du poème. Dans le prologue du chant $\mathrm{V}$, le poète célèbre la gloire d'Épicure, que ses découvertes placent au rang des dieux :

On dit que Cérès fit connaitre le blé aux hommes et Liber la liqueur née du jus de la vigne.

Pourtant la vie pouvait demeurer sans ces biens qui de certains peuples semblent encore ignorés. Mais on ne saurait vivre heureux sans un cœur pur.

Aussi est-il plus digne de nous paraître un dieu

celui dont les doctrines parmi les grandes nations

divulgua les douces consolations de la vie

qui de nos jours encore apaisent les esprits ${ }^{59}$.

Namque Ceres fertur fruges Liberque liquoris

uitigenti laticem mortalibus instituisse ;

at bene non poterat sine puro pectore uiui ;

quo magis hic merito nobis deus esse uidetur,

ex quo nunc etiam per magnas didita gentis

dulcia permulcent animos solacia uitae. (DRN, V, 18-22)

La comparaison avec Cérès et Liber permet de souligner la supériorité d'Épicure, dont la doctrine console des maux de la vie. Dans les vers qui suivent (V, 24-42), une comparaison avec les travaux d'Hercule, héros stoïcien par excellence, souligne les enjeux de la victoire remportée par Épicure, qui par la vertu de sa seule parole, libéra l'homme de ses tourments intérieurs. Et Lucrèce d'insister tout particulièrement sur la puissance du verbe épicurien ${ }^{60}$, dont il revendique aussi la maîtrise pour lui-même ${ }^{61}$.

Cette idée est réaffirmée et précisée dans le prologue du chant VI : le plus grand mérite attribué à la ville d'Athènes est d'avoir donné naissance au héros Épicure, offrant ainsi de «douces consolations » aux malheureux mortels (4-5). Lucrèce lie explicitement l'efficacité des consolations prodiguées par le fondateur du Jardin au caractère véridique de sa parole ${ }^{62}$ :

Par ses paroles de vérité purifiant donc les cœurs,

Il fixa une borne au désir comme à la crainte

Et du bien suprême auquel nous aspirons tous

Il exposa la nature et montra le chemin [...]

Veridicis igitur purgauit pectora dictis

et finem statuit cuppedinis atque timoris

exposuitque bonum summum, quo tendimus omnes,

quid foret, atque uiam monstrauit, [...] (DRN, V, 24-27)

19 L'action consolatrice de la vérité est assimilée à une purge d'ordre cognitif : pour apaiser les âmes et évacuer les soucis qui déchirent les cœurs ${ }^{63}$, le philosophe doit substituer à un ensemble d'opinions erronées et sans fondement scientifique, le système salvateur de la vraie doctrine, uera ratio.

Les emplois du substantif solacium, qui est étroitement associé à la geste d'Épicure, soulignent l'importance attribuée à la consolation dans la doctrine du Jardin. En valorisant dans les prologues la figure d'un Épicure consolateur, qui a généreusement 
dispensé ses remèdes à une humanité souffrante, Lucrèce rattache son poème à la tradition des écrits philosophiques consolatoires. Cette orientation apparaitt également dans la structure de l'argumentation lucrétienne, qui associe prophylaxie et thérapie.

\section{Thérapie et prophylaxie}

21 L'objectif de Lucrèce est de débarrasser l'esprit des troubles (curae) suscités par les désirs vains et par la crainte, deux affections qui portent sur l'avenir. Or, à la différence du désir, qui n'est pas mauvais en lui-même et doit être évalué en fonction de son objet $^{64}$, la crainte représente une affection totalement néfaste dont les causes et les effets sont décrits dans le prologue du chant III (31-97). Cette affection correspond à une projection dans l'avenir, qui détourne l'âme des joies réelles et provoque une angoisse pathologique ${ }^{65}$. Cependant, la crainte de la mort est particulièrement néfaste car elle a pour effet de plonger les mortels dans un torrent de désirs insatiables: croyant vainement se protéger de la mort en accumulant richesses et honneurs, les hommes s'adonnent à l'ambition et la cupidité, qui sont des désirs voués à l'insatisfaction et incompatibles avec l'ataraxie (III, 65-67). À cette souffrance vient s'ajouter la crainte du châtiment, car le crime est le compagnon inévitable de l'avidité. L'image de la mort entraine donc un concert de maux, qui détournent l'homme du bonheur présent pour le projeter dans le temps illusoire des passions ${ }^{66}$. C'est pourquoi, Lucrèce, avant de s'attaquer à la crainte de la mort, dénonce avec véhémence l'erreur qui abuse ses semblables. Égarés par une crainte injustifiée et croyant éviter le pire, ils se privent du bien suprême et peuvent aller jusqu'à se suicider, oubliant que la source de leurs angoisses est cette crainte elle-même (DRN, III, 79-82).

L'objectif prioritaire de la thérapie lucrétienne sera donc de combattre la crainte de la mort, qui se trouve à l'origine de tous les troubles de l'âme ${ }^{67}$. Il faut noter que cette hiérarchisation n'est pas le fait des seuls épicuriens. On retrouve la même conception chez l'académicien Cicéron, qui, d'une part, voit dans le chagrin la source et l'origine de tous les maux et, d'autre part, considère le chagrin lié au deuil comme la forme la plus redoutable ${ }^{68}$. Cette priorisation apparaît notamment dans la structure même des Tusculanes, qui présentent la consolatio mortis, conduite dans la première conférence, comme un paradigme de toutes les consolations particulières, qu'elles concernent l'exil, la destruction de la patrie, la paralysie, la cécité. Et Cicéron de conclure, en se référant à l'analogie entre philosophie et médecine, sur l'universalité de la consolation philosophique, qui « en supprimant le chagrin en général, supprime du même coup les idées fausses quelle que soit leur origine ${ }^{69} »$. En montrant, comme le fera Cicéron, que la mort n'est pas un mal et en donnant les moyens de surmonter les troubles de l'âme qu'elle produit, le philosophe épicurien fait donc œuvre de consolation non seulement relativement à la mort elle-même mais pour tous les maux de la vie.

On pourra objecter que Lucrèce s'attache plus à soulager l'angoisse liée à la pensée de la mort qu'à apaiser le chagrin provoqué par le deuil ${ }^{70}$. Cependant, réfuter la crainte de la mort permet de prévenir l'angoisse du mourant et le désespoir de ses proches: bien que leur ancrage temporel soit différent, les discours ante et post mortem visent au même résultat à savoir la tranquillité de l'âme. Ce lien fonctionnel est clairement mis en évidence par les occurrences du substantif latin metus, la crainte, qui, dans le $D e$ rerum natura, est très fréquemment associé au chagrin et au deuil ${ }^{71}$. Dans la mesure où les maladies de l'âme ont une source commune et sont étroitement interdépendantes, 
la perspective temporelle adoptée - a priori ou a posteriori - ne fait guère de différence au plan argumentatif ${ }^{2}$. De plus, Lucrèce n'ignore pas totalement le point de vue de l'endeuillé, auquel il donne provisoirement la parole dans un passage fortement influencé par la diatribe ${ }^{73}$.

\section{Scène de consolation}

Face à la mort d'un père de famille, l'un de ses proches, possiblement sa veuve, se lamente en se référant au mort lui-même, qui, même s'il ne souffre pas, ne peut plus jouir des biens de la vie (DRN, III, 894-930). À cette déploration qui constitue une caricature des formules de deuil usuelles, Lucrèce répond que le mort ne ressent aucune frustration de ces avantages. L'endeuillé(e) enchaîne alors en se lamentant sur son propre sort :

Toi, du moins, tel que tu es dans la mort endormi, tel à jamais tu seras, exempt de toutes souffrances. Mais nous, près de tes cendres, de ce bûcher horrible, Inlassablement nous pleurons, et ce chagrin éternel, aucun jour à nos cœurs ne l'arrachera. " Demandons-nous alors ce qui a tant d'amertume, si la chose revient au calme et au sommeil, pour que l'on se consume en un deuil éternel. $\mathrm{Tu}$ quidem ut es leto sopitus, sic eris aeui quod super est cunctis priuatus doloribus aegris ; at nos horrifico cinefactum te prope busto insatiabiliter defleuimus, aeternumque nulla dies nobis maerorem e pectore demet ». Illud ab hoc igitur quaerendum est, quid sit amari tanto opere, ad somnum si res redit atque quietem, cur quisquam aeterno possit tabescere luctu. (DRN, III 904-911)

Cette lamentation fictive est structurée par la division traditionnelle entre la condition du défunt, condicio mortui, et celle de l'endeuillé, condicio maerentis, qui est bien attestée dans le corpus consolatoire ${ }^{74}$. En l'occurrence, l'argument majeur de l'insensibilité, qui est intégré à la plainte, fonde en raison l'interrogation rhétorique de Lucrèce: la pensée que le défunt lui-même ne soit plus sujet au regret ne suffit-elle pas à limiter considérablement la peine de ses proches ? Aussi, faut-il prêter attention à l'insistance de Lucrèce sur l'adjectif aeternus relayée par les formes adverbiales tanto opere et insatiabiliter. Ces éléments lexicaux traduisent certes l'ironie de Lucrèce face à une tristesse qui se croit éternelle quand tout ce qui est humain s'inscrit dans un temps limité. Cependant, ils montrent également que Lucrèce entend surtout dénoncer le caractère excessif et interminable du chagrin, auquel le deuil donne traditionnellement lieu, mais qu'il n'exige pas de ses lecteurs une totale insensibilité face à la mort ${ }^{75}$. Le caractère naturel du chagrin causé par la perte d'un proche est du reste confirmé, dans un tout autre contexte argumentatif, par l'évocation pathétique d'une vache que rien ne peut consoler de sa peine, suite à la perte de son veau sacrifié ${ }^{76}$. Le même type de lecture peut être appliqué au discours de la Nature qui, en dépit de sa sévérité, présente également des modalisateurs suggérant une certaine tolérance à l'égard des réactions émotionnelles face à la mort ${ }^{77}$. Ce sont les comportements déraisonnables, dont Niobé et Artémise constituent des illustrations paradigmatiques ${ }^{78}$, que condamne Lucrèce. Or ces états pathologiques trouvent leur origine dans des opinions erronées et un vain désir d'immortalité que l'épicurisme se propose précisément de rectifier. Tout en 
s'inscrivant dans la doctrine du Jardin, les reproches formulés par le philosophe latin s'accordent par conséquent aux devoirs traditionnellement assignés aux consolateurs, qui, s'ils ne peuvent supprimer radicalement le chagrin, doivent s'efforcer de le diminuer voire de l'empêcher de s'étendre ${ }^{79}$. Quant aux réprimandes adressées à tous ceux qui se lamentent inconsidérément face à la mort, elles renvoient à la composante exhortative des consolations, qui font généralement appel au courage et à la fermeté d'âme du destinataire ${ }^{80}$. De son côté, Lucrèce exhorte ses lecteurs à mettre en œuvre la force de la raison pour vivre en conformité avec les principes de la « vraie doctrine ».

En conclusion, il apparaît que le De rerum natura, qui entretient des liens complexes avec la tradition de la consolation, entre dans la catégorie des méta-consolations. Plaçant la crainte de la mort au cœur de son dispositif thérapeutique, Lucrèce est amené à faire fond sur les topiques, les méthodes et les arguments de la consolation qu'il met au service de son projet philosophique. Inversement, il intègre à son exposé un scénario de consolation visant à stigmatiser et à rectifier les erreurs de jugement relatives à la mort et à la condition humaine. L'argument central de l'insensibilité posthume se trouve ainsi amplifié par son inscription dans le champ de l'expérience et des pratiques sociales. En ce sens, le poème de Lucrèce définit les conditions de possibilité d'une consolation épicurienne et confirme la thèse de $\mathrm{M}$. Fœssel, selon laquelle « il y a une rationalité propre à l'acte de consoler ${ }^{81}$ ». Pourtant, la contradiction entre philosophie et consolation demeure irréductible car le besoin de réconfort marque en quelque sorte les limites du champ philosophique. Le sage, qui s'est préparé au malheur par l'exercice de la raison, ne devrait avoir nul besoin d'être consolé face à l'adversité, contrairement à la majorité des hommes, qui n'ont pas voulu ou pas su mettre en pratique les leçons de la philosophie. C'est à ces mortels insensés et imprévoyants, que Lucrèce propose une thérapie préventive, qui les mettra en capacité de surmonter sereinement les épreuves les plus terribles. En ce sens, le De rerum natura se veut une consolation radicale, définitive et universelle.

\section{NOTES}

1. P. Vallette, «Lucrèce et la diatribe ", Revue des Études Anciennes, 42, 1940, Mélanges d'études anciennes offerts à Georges Radet, p. 532-541, citation p. 534-535; doi : 10.3406/rea.1940.3137 http://www.persee.fr/doc/rea_0035-2004_1940_num_42_1_3137.

2. Sur la valorisation de la raison dans le De rerum natura (désormais $D R N$ ), voir, par exemple, DRN, I, 146-150 ; II, 59-61 ; III, 91-93 ; VI, 39-41. La doctrine épicurienne est désignée par l'expression uera ratio (DRN, II, 1023). Pour une vision d'ensemble sur la structure de l'argumentation lucrétienne, voir P. Boyancé, Lucrèce et l'épicurisme, Paris, PUF, 1963.

3. Lucrèce, DRN, II, 1-61; III, 1-30.

4. Voir, notamment, C. J. Classen, "Poetry and Rhetoric in Lucretius", Transactions and Proceedings of the American Philological Association, 99, 1968, p. 77-118 ; P. H. Schrijvers, Horror ac diuina uoluptas, Étude sur la poétique et la poésie de Lucrèce, Amsterdam, A. M. Hakkert, 1970 ; G. Calboli, "Lucrezio e la retorica ", Paideia, 58, 2003, p. 186-206; P. H. Schrijvers, «Propagandistic strategies in Lucretius' De rerum natura», dans A. Harder, A. Macdonald \& 
G. Reinink éd., Calliope's Classroom. Studies in Didactic Poetry from Antiquity to the Renaissance, Louvain/Paris, Peeters, 2007, p. 49-69 ; D. Markovic, The Rhetoric of Explanation in Lucretius De rerum natura, Leyde, J. Brill, 2008.

5. M. Fœssel, Le temps de la consolation, Paris, Seuil, 2015, p. 10-13.

6. Concernant l'influence de la rhétorique sur le chant III, voir E. Rand, «La composition rhétorique du troisième livre de Lucrèce ", Revue de philologie, de littérature et d'histoire Anciennes, 8, 1934, p. 243-266 ; C. Rambaux, «La logique de l'argumentation dans De rerum natura III, 830-1094», Revue des Études Latines, 58, 1980, p. 201-219.

7. Voir J. Salem, Lucrèce et l'éthique. La mort n'est rien pour nous, Paris, Vrin, 1990, p. 121-167.

8. C. Alonso Del Real dir., Consolatio. Nueve estudios, Pampelune, Ediciones Universidad de Navarra, 2001; F. Lillo Redonet, Palabras contra el dolor. La consolacion filosofica latina de Ciceron a Fronton, Madrid, Ediciones Clásicas, 2001 ; H. Baltussen dir., Greek and Roman Consolations. Eight Studies of a Tradition and its Afterlife, Swansea, The Classical Press of Wales, 2013.

9. D. Konstan, «Lucretius and the Epicurean Attitude towards Grief », dans D. Lehoux et al. dir., Lucretius : Poetry, Philosophy, Science, Oxford, Oxford University Press, 2013, p. 193-210.

10. Voir notamment R. Sorabji, Emotion and Peace of Mind, Oxford, Oxford University Press, 2002 ; D. Konstan, The Emotions of the Ancient Greeks. Studies in Aristotle and Classical Literature, Toronto, University of Toronto Press, 2006 ; D. Armstrong, « Be angry and sin not. Philodemus versus the Stoics on natural bites and natural emotions", dans D. F. Fitzgerald (dir.), Passions and moral Progress in Graeco-Roman Thought, Londres/New York, Routlege, 2008, p. 79-121; D. Konstan, "Epicurean "Passion" and the Good Life ", dans B. Reis dir., The Virtuous Life in Greek Ethics, Cambridge, Cambridge University Press, 2006, p. 194-205.

11. Concernant l'influence de la diatribe sur le De rerum natura, voir E. J. Kenney, Lucretius. De Rerum Natura Book III, Cambridge, Cambridge University Press, 1971, p.17-20 ; B. Wallach, Lucretius and the Diatribe against the Fear of Death, Leyde, J. Brill, 1976, p. 11-109 ; J. Salem, Lucrèce et l'éthique, op. cit., p. 117-119.

12. Il est convenu d'appliquer ce terme à des écrits moraux, plus ou moins influencés par la tradition cynique, et caractérisés "par une situation d'énonciation marquée par le schème scolaire maître-disciple». Sur cette (re)définition de la diatribe en contexte d'énonciation pédagogique, voir P. P. Fuentes Gonzalez, Les diatribes de Télès, Paris, Vrin, 1998, p. 44-78, citation p. 55.

13. Voir DRN, III, 894-911 (lamentations des endeuillés) et 931-977 (prosopopée de la Nature).

14. Voir P. M. Brown, Lucretius De Rerum Natura III, with an Introduction, Text, Translation \& Commentary, Warminster, Aris \& Phillips Ltd, 1997, p.190-203 ; P. P. Fuentes Gonzalez, Les diatribes de Télès, op. cit., p. 185.

15. Voir Ps. Plutarque, Vie des X orateurs grecs, Antiphon, 833d. Sur ce logographe, parfois identifié à Antiphon de Rhamnonte, qui participa à la révolution oligarchique de 411, et/ou à Antiphon le Sophiste, auteur des traités Sur la vérité et Sur la Concorde, conservés à l'état de fragments papyrologiques, voir M. Narcy, « Antiphon d'Athènes RE 15 », dans R. Goulet dir., Dictionnaire des philosophes antiques, I, Paris, CNRS Éditions, 1989, p. 225-244 ; A. Hourcade, Antiphon d'Athènes. Une pensée de l'individu, Paris, Ousia, 2001. Pour le texte, voir les éditions de L.Gernet, Antiphon, Discours. Fragments d'Antiphon, Paris, Les Belles Lettres (CUF), 1923 et de G. J. Pendrick, Antiphon the Sophist, The Fragments, Cambridge (UK), University Press, 2002.

16. Parmi les consolations perdues en totalité ou partiellement, dont l'existence nous est connue de façon indirecte, on peut citer notamment le Peri penthous (Sur le deuil) de Crantor (voir infra note 36), la consolation composée par Clitomaque suite à la destruction de Carthage en 146 (voir Cicéron, Tusculanes, III, 54), la Consolatio ad se que Cicéron s'adressa à lui-même après la mort de sa fille Tullia en 45 (Cicéron, Tusculanes, I, 65-67; 76; 115 ; III, 70 ; 76 ; IV, 63), celle qu'Arius Didyme adressa à Livie (Sénèque, Consolation à Marcia, 5, 2). 
17. Sur le corpus cicéronien des consolations épistolaires, voir F. Lillo Redonet, Palabras contra el dolor, op. cit., p. 110-113. Sur le corpus sénéquien : voir Sénèque, Lettres à Lucilius, 63 ; 93 ; 99; Consolation à Marcia (sur la mort de son fils); Consolation à Helvia (sur l'exil de son fils Sénèque) ; Consolation à Polybius (sur la mort de son fils). Sur le corpus de Plutarque, voir Plutarque, Consolation à sa femme, sur la mort de leur fille Timoxéna. Sur la Consolation à Apollonios et les problèmes d'attribution qu'elle pose, voir M. Cannatà Fera, «Plutarco e la Consolatio ad Apollonium », Messana, 15, 1993, p. 121-153. Enfin sur le corpus de Pline le Jeune : voir la lettre (IX, 9) que Pline le Jeune adresse à un certain Colon à l'occasion de la mort de leur ami commun, Pompeius Quitianus. La visée consolatoire de cette lettre a été récemment étudiée par S. Audano, «Memoria e consolatio in Plinio il Giovane. Per una lettura di ep. 9, 9 », Vichiana, 52, 1-2, 2015, p. 59-70.

18. Pour Catulle : voir Catulle, c. 96. Sur ce poème adressé à Licinius Calvus suite au décès de sa femme Quintilia, voir F. Bellandi, «Calvo e Quintilia e l'esegesi del c. 96 di Catullo », MD, 55, 2005, p. 123-142. Pour Horace : voir, par exemple, les Odes I, 24, adressée à Virgile pour la mort de l'ami commun Quintilius Varus, et II, 9, adressée à C. Valgius Rufus pour la mort de Mystès. Sur ces consolations horatiennes, voir A. Setaioli, « Le Virgile d'Horace », Prometheus, 32, 2006, p. 161-184, particulièrement p. 177-181.

19. Sur la Consolation à Livie, attribuée à Ovide, voir J. Amat, Consolation à Livie. Élégies à Mécène. Bucoliques d'Einsiedeln, Paris, Les Belles Lettres (CUF), 1997, p.19-43, qui défend l'attribution à Ovide.

20. Voir Cicéron, De l'orateur, II, 50 ; 64 ; III, 118.

21. Voir R. Kassel, Untersuchungen zur griechischen und römischen Konsolationsliteratur, Munich, Beck, 1958, p. 40-48 et A. Setaioli, « Il destino dell'anima nella letteratura consolatoria pagana », dans C. Alonso Del Real (dir.), Consolatio, op. cit., p. 31-67 (p. 32).

22. Voir le Pseudo-Denys, Ars rhetorica, VI, 4-6, dans Dionysii Halicarnasei quae exstant, éd. H. Usener et R. Radermacher, Stuttgart, Teubner, 1965, II, p. 281, 1-283, 19 ; Ménandre le Rhéteur, II, 9, 413-415, dans Menander Rhetor, éd., trad., comm. par D. A. Russell et N. G. Wilson, Oxford, Clarendon Press, 1981, p. 161-165.

23. Ps. Démétrios de Phalère, Types épistolaires, 5, traduction P.-L. Malosse, Lettres pour toutes circonstances, Paris, Les Belles Lettres, 2004.

24. Définition moderne donnée par L. Pernot, La rhétorique dans l'Antiquité, Paris, Librairie Générale française, 2000, p. 261.

25. Le quatrième livre de ce traité était consacré à la thérapie des passions. Pour une reconstitution et une analyse du traité, dont il ne reste que des fragments, voir T. Tieleman, Chrysippus, On affections, Leyde/Boston, Brill, 2003.

26. Voir Cicéron, Des Termes extrêmes des biens et des maux, IV, 23.

27. Sur la dimension consolatoire des Tusculanes, qui se présentent comme une thérapie progressive de l'âme, voir A. Michel, « Rhétorique et philosophie dans les Tusculanes ", Revue des Études Latines, 39, 1961, p. 158-171 ; Id., " Rhétorique et maladie de l'âme. Cicéron et la consolation des passions », Littérature, médecine et sociétés, 1983, p. 11-22 ; M. Rodríguez Pantoja, « La consolatio y las Disputationes Tusculanae de Cicerón ", dans C. Alonso Del Real dir., Consolatio, op. cit., p. 69-97 ; B. Koch, Philosophie als Medizin für die Seele. Untersuchungen zu Ciceros Tusculanae Disputationes, Stuttgart, Franz Steiner Verlag, 2006 et S. Luciani, Temps et éternité dans l'œuvre philosophique de Cicéron, Paris, PUPS, 2011, p. 317-348.

28. R. Kassel, Untersuchungen zur griechischen und römischen KonsolationsLiteratur, op. cit., p. 4-12.

29. Cette distinction est formulée par Cicéron dans les Tusculanes (III, 81, traduction J. Humbert, Paris, Les Belles Lettres, CUF, 1968), qui oppose au deuil, forme la plus redoutable de chagrin, d'autres types de maux plus supportables: "Pour la pauvreté en effet, pour l'existence dépourvue d'honneur et de gloire, nous avons des arguments topiques qui sont d'usage courant sur ces sujets : il existe des cours spéciaux consacrés à l'exil, à la destruction de la patrie, à 
l'esclavage, à la paralysie, à la cécité, à tous les accidents auxquels s'applique usuellement le nom de calamité. Chacune de ces matières-là est l'objet de cours et d'ouvrages particuliers chez les Grecs. »

30. Voir Cicéron, Tusculanes, III, 74-79.

31. D. Scourfield, "Towards a Genre of Consolation », dans H. Baltussen éd., Greek and Roman Consolations, op. cit., p. 1-36.

32. Sur ce philosophe académicien du début du III ${ }^{\mathrm{e}}$ siècle, voir T. Dorandi, "Crantor de Soles ", dans R. Goulet dir., Dictionnaire des Philosophes antiques, II, Paris, CNRS Éditions, 1994, p. 482-483. Le traité, aujourd'hui perdu, qu'il avait adressé à son ami Hippoclès pour le consoler de la mort de ses enfants fut explicitement pris pour modèle par Cicéron dans sa Consolation (voir Cicéron, Académiques, II, 135 ; Tusculanes, I, 113-115 ; III, 71 ; Pline, Histoire naturelle, Praef. 22 ; Jérôme, Lettres, 60, 5) et par le Pseudo-Plutarque (Consolation à Apollonios, 102c-d; 104c; 114c-e). Les fragments ont été rassemblés par H. Mette, «Zwei Akademiker heute: Krantor von Soli und Arkesilaos von Pitane », Lustrum, 26, 1984, p. 7-93.

33. Voir, par exemple, Cicéron, Tusculanes, IV, 82 et III, $68 ; 81$.

34. Sur la dimension thérapeutique de la philosophie hellénistique, voir A. J. Voelke, La philosophie comme thérapie de l'âme. Études de philosophie hellénistique, Fribourg, Éditions Universitaires / Paris, Le Cerf, 1993.

35. Plutarque, Qu'il n'est pas non plus possible de vivre plaisamment en suivant Épicure, 1101a-b.

36. Sur la métriopathie communément attribuée aux péripatéticiens et au philosophe académicien Crantor, voir Cicéron, Lettres à Titus Pomponius Atticus, XII, 14, 3; Tusculanes, IV, 38-47 ; Académiques, I, 38-39 ; II, 135.

37. R. Kassel, Untersuchungen zur griechischen und römischen KonsolationsLiteratur, op. cit., p. 29-32.

38. Les leçons de l'éthique épicurienne sont rassemblées dans le "quadruple remède" (tetrapharmakos), qui correspond aux quatre premières Maximes Capitales d'Épicure : Dieu n'est pas à craindre; la mort ne nous concerne pas; le bien est facile à obtenir; le mal est facile à supporter. Voir Philodème, Aux amis de l'École, dans Papyrus d'Herculanum 1005, v. 9-13 Angeli (Long \& Sedley, I, 25 J).

39. Traduction P.-M. Morel, Épicure. Lettres, maximes et autres textes, Paris, GF Flammarion, 2011.

40. Plutarque, Qu'il n'est pas non plus possible de vivre plaisamment en suivant Épicure, 1101a-b.

41. Diogène Laërce, Vies et doctrines des philosophes illustres, $\mathrm{X}, 117-120$.

42. Sénèque, Lettres à Lucilius, 99, 25 ; Plutarque, Qu'il n'est pas non plus possible de vivre plaisamment en suivant Épicure, 1097e-f.

43. D. Konstan, «The Grieving Self. Reflections on Lucian's On Mourning and the consolatory tradition », op. cit., p. 139-152 (p. 147).

44. Voir supra, note 36.

45. Voir Épicure, Lettre à Ménécée, 125 : «Ainsi, le plus effroyable des maux, la mort, n'est rien pour nous, étant donné, précisément, que quand nous sommes, la mort n'est pas présente; et que, quand la mort est présente, alors nous ne sommes pas. Elle n'est donc ni pour les vivants ni pour ceux qui sont morts, étant donné, précisément, qu'elle n'est rien pour les premiers et que les seconds ne sont plus », traduction P. M. Morel, Paris, GF Flammarion, 2011.

46. Ph. Mitsis, L'Éthique d'Épicure. Les plaisirs de l'invulnérabilité, traduction française A. Gigandet, Paris, Classiques Garnier, 2014, p. 23.

47. Le traité De morte de Philodème comportait quatre livres, dont il ne nous reste que la fin du quatrième (Papyrus d'Herculanum 1050), voir Les Épicuriens, D. Delattre et J. Pigeaud dir., Paris, Gallimard, 2010, p. 631-633 et notice p. 1276-1278, traduction D. Delattre et V. Tsouna.

48. Sur la chronologie de Philodème, né vers 110 et mort vers 40, voir l'introduction de D. Delattre, dans Philodème, Sur la musique, éd. D. Delattre, Paris, Les Belles Lettres, (CUF), 2007, p. XI-XXII. Sur celle de Lucrèce, né entre 96 et 94 et mort entre 53 et 51, voir J. Kany-Turpin, 
«Lucretius Carus (T.-)», L 73, dans R. Goulet dir., Dictionnaire des Philosophes Antiques, IV, Paris, CNRS Éditions, 2005, p. 174-191.

49. Voir notamment C. Bailey, De rerum natura Libri sex, Oxford, Clarendon, 1947, tome 2, p. 1143 ; E. J. Kenney, Lucretius. De rerum natura Book III, op. cit., p. 212 ; A. R. Sharrock, « The philosopher and the mother cow : towards a gendered reading of Lucretius De rerum natura ", dans V. Zajko \& M. Leonard dir., Laughing with Medusa : Classical Myth \& Feminist Thought, Oxford, Oxford University Press, 2006, p. 253-274 (p. 267) ; A. D. Morrisson, « Nihil mors est ad nos? Iphianassa, the Athenian Plague, and Epicurean Views of Death ", dans D. Lehoux et al. dir., Lucretius : Poetry, Philosophy, Science, op. cit., p. 211-232, particulièrement p. 229-231.

50. Voir J. Fish, «Is death nothing to Horace? A brief comparison with Philodemus and Lucretius ", Cronache Ercolanesi, 28, 1998, p. 99-104 ; J. Warren, Facing Death. Epicurus and his critics, Oxford, Clarendon Press, 2004, p. 143-159 et 193-195; V. Tsouna McKirahan, The Ethics of Philodemus, Oxford, Oxford University Press, 2007; D. Armstrong, «Be angry and sin not. Philodemus versus the Stoics on natural bites and natural emotions", op. cit., p. 79-121; R. K. Sanders, «Philodemus and the fear of premature death", dans J. Fish \& K. R. Sanders éd., Epicurus and the epicurean tradition, Cambridge, Cambridge University Press, 2011, p. 211-234 ; D. Konstan, "The Grieving Self. Reflections on Lucian's On Mourning and the consolatory tradition », op. cit., p. 146-148; id., "Lucretius and the Epicurean attitude towards grief », op. cit., p. 194-209.

51. D. Armstrong, «Be angry and sin not. Philodemus versus the Stoics on natural bites and natural emotions $»$, op. cit.

52. Voir supra, notes 44 et 46.

53. Pour une analyse détaillée de ces vers, voir T. Reinhardt, "The Speech of Nature in Lucretius' De Rerum Natura 3.931-71", The Classical Quarterly, N.S. 52.1, 2002, p. 291-304 et S. Luciani, «Lucrèce et la voix de la Nature », Vita Latina, 183-184, mars 2011, p. 205-218, accessible en ligne http://www.persee.fr/doc/vita_0042-7306_2011_num_183_1_1722.

54. Repris par iure increpet au vers 963. La Nature s'adresse au mortel comme à un inférieur qu'elle rappelle énergiquement à la raison. Loin d'exclure la consolation, la réprimande en est ici l'instrument efficace. Voir, dans l'ATELIER de ce numéro, l'emploi du même increpet par Vossius (Rhetorices contractae..., II, 24, § 8) : Major utrisque aget liberius, adeo ut, siquis immoderatius dolorem ferat, etiam increpet graviter.

55. Les traductions du De rerum natura sont empruntées à J. Kany-Turpin, Paris, Aubier, 1993. L'idée selon laquelle les désirs sans objet nuisent au plaisir présent en projetant le sujet dans l'avenir figure chez Épicure, Sentences Vaticanes 35 : « Il ne faut pas gâter les choses présentes par le désir des absentes, mais considérer que celles-là mêmes étaient appelées de nos vœux ».

56. Voir Ménandre le Rhéteur, II, 9, 413. Lucrèce fait preuve de la même impatience dans plusieurs autres passages (III, 916-917 ; 933-934; 1045-1052).

57. Voir A. D. Morrisson, « Nihil mors est ad nos? Iphianassa, the Athenian Plague, and Epicurean Views of Death », op. cit., p. 229.

58. Voir Catulle, c. 64, 182 ; Horace, Satires, I, 6, 128 ; Ovide, Pontiques I, 9, 19 ; III, 3, 77 ; III, 6, 13 ; Métamorphoses I, 359, 577...

59. Voir aussi DRN, VI, 1-25.

60. Voir DRN, V, 50 : «expulerit dictis, non armis »: «il les chassa < de notre âme> par ses seules paroles, et non par les armes ».

61. Voir DRN, V, 114: «multa tibi expediam doctis solacia dictis»: «mes savants discours t'apporteront mainte consolation ».

62. Voir DRN, VI, 6: «omnia ueridico qui quondam ex ore profudit»: «<le héros> dont la bouche véridique révéla toutes choses ».

63. DRN, V, 43-48. 
64. Sur la classification épicurienne des désirs, voir Épicure, Lettre à Ménécée, 127-128; Maximes Capitales, 29 et 30.

65. Voir, par exemple, DRN, II, 47 ; III, 825-826.

66. Sur les liens entre temps et passions chez Lucrèce, je me permets de renvoyer à $\mathrm{S}$. Luciani, L'éclair immobile dans la plaine. Philosophie et poétique du temps chez Lucrèce, Paris, Peeters, 2000, p. 191-214.

67. Voir aussi DRN, III, 37-43.

68. Voir Cicéron, Tusculanes, IV, 82 : fons miserarium et caput ; III, 68 et 81.

69. Cicéron, Tusculanes, III, 82, traduction J. Humbert, Paris, Les Belles Lettres (CUF), 1931.

70. Cette perspective prophylactique est clairement formulée par Épicure, Lettre à Ménécée, 125 : «Il n'y a rien de terrible dans le fait de vivre, lorsqu'on a réellement saisi que dans le fait de pas vivre il n'y a rien de terrible (aussi est-il stupide celui qui dit craindre la mort non pour la peine que sa présence lui causera, mais pour celle que sa perspective lui cause ; car ce dont la présence ne nous tourmente pas ne cause qu'une peine sans fondement lorsqu'on l'attend). »

71. Voir DRN, IV, 1183 ; DRN, IV, III, 459-461 ; DRN, III, 902-903.

72. L'argument apparaît explicitement chez Cicéron, Tusculanes, III, 14 : « Or celui qui est sujet au chagrin l'est aussi à la crainte car les événements dont la présence provoque le chagrin, sont aussi ceux dont nous craignons l'imminence et la venue ».

73. Voir supra, notes 11,12 et 13.

74. Voir, par exemple, la lettre de consolation adressée par Cicéron à Titius (Lettres familières, V, $14,4)$ ou la consolation adressée à Cicéron par Sulpicius Rufus suite à la mort de sa fille Tullia (Lettres familières, IV, 5, 2-3).

75. Ce point a été souligné par D. Konstan, «Lucretius and the Epicurean attitude towards grief ", op. cit., p. 206.

76. Voir DRN, III, 355-366.

77. Voir notamment les adverbes tanto opere (à ce point) et nimis (trop) en DRN, III, 933-934, le comparatif amplius aequo (plus que de raison), en DRN, III, 952-953 et l'analyse de J. Fish, « Is death nothing to Horace? A brief comparaison with Philodemus and Lucretius », op. cit., p. 101.

78. Sur ces figures d'inconsolables, voir Cicéron, Tusculanes, III, $63 ; 75$ et les analyses de M. Fœssel, Le temps de la consolation, op. cit., p. 99-102.

79. Voir Cicéron, Lettres familières, V, 14, 1 ; Lettres à Marcus Junius Brutus, I, 9 ; Tusculanes, III, 75 ; Horace, Odes, II, 9 ; Plutarque, Consolation à sa femme, 2 ; Consolation à Apollonios, 3.

80. Voir, par exemple, Cicéron, Lettres familières, IV, 5,$5 ; \mathrm{V}, 14,5$; Lettres à Marcus Junius Brutus, I, 9 ; Sénèque, Consolation à Marcia, I, 1-5; Consolation à Helvia, XVI, 1-7, Consolation à Polybius, VI, 1-5.

81. M. Fœssel, Le temps de la consolation, op. cit., p. 35.

\section{AUTEUR}

\section{SABINE LUCIANI}

Aix-Marseille Université, CNRS / TDMAM 